\title{
Tesis de Maestría \\ Maestría en Docencia de la Química \\ Departamento de Química
}

\section{Enseñanza aprendizaje por investigación de los cambios químicos a través de la resolución de problemas}

Nohora Liliana Burgos Leguizamón

Descripción: este trabajo investigativo se desarrolló con estudiantes de grado décimo del Colegio Emilio Valenzuela, ubicado en la localidad de Suba, en Bogotá. Para la implementación de la estrategia se tuvieron en cuenta dos aspectos fundamentales: la construcción progresiva del concepto cambio químico y la implementación del modelo didáctico enseñanza-aprendizaje por investigación centrado en la resolución de problemas. La evaluación de la estrategia se realizó con base en los cambios generados en los estudiantes a nivel conceptual, metodológico y actitudinal.

Metodología: la investigación que se realizó es de carácter experimental y se desarrolló a través de las siguientes unidades de observación:

1. Diagnóstico y aplicación de instrumentos para determinar el estado inicial de los estudiantes en cuanto a conceptos, actitudes y metodología.

2. Diseño de la estrategia didáctica de acuerdo con los supuestos teóricos y con los resultados del diagnóstico.

3. Aplicación de la estrategia metodológica basada en la enseñanza-aprendizaje por investigación centrada en la resolución de problemas, en los cambios químicos y apoyada en los módulos didácticos diseñados para tal fin.

4. Aplicación de instrumentos para identificar al final del proceso el cambio conceptual, metodológico y actitudinal.

Conclusiones: la implementación de la estrategia generó un cambio conceptual acerca de los cambios químicos. Inicialmente, sólo un $8 \%$ de la población se encontraba en los niveles medio alto y alto, mientras que al final, la mayoría de los estudiantes $(84 \%)$ se encontraban en estos niveles, demostrándose así que los estudiantes mejoraron su conocimiento sobre los cambios químicos.

Se observó que inicialmente, en el aspecto metodológico, solamente el $3 \%$ de la población se encontraba en los niveles medio alto y alto, mientras que al final de la estrategia la mayoría de los estudiantes $(85 \%)$ alcanzo estos niveles, lo que indica que la estrategia generó un cambio metodológico en la resolución de problemas, en el que los estudiantes lograron desarrollar habilidades y capacidades propias de las ciencias.

Con el desarrollo de la estrategia los estudiantes mejoraron sus actitudes hacia la clase de química. Inicialmente, el $67 \%$ de los estudiantes se encontraban en el polo positivo de la escala (1,2 y 3 ), mientras que al final de la estrategia aumentó al $89 \%$. 


\section{Contribución del software educativo al aprendizaje significativo de los conceptos punto de ebullición y densidad en estudiantes de grado décimo}

\section{Martha Elizabeth Villarreal Hernández}

Descripción: en esta investigación se ha incorporado un software educativo diseñado con base en los principios del modelo de enseñanza y aprendizaje por investigación para la enseñanza de los conceptos de punto de ebullición y densidad. Se expone cómo un programa guía que contiene actividades de conflicto cognitivo, apoyadas con imágenes, animaciones y simulaciones de laboratorio virtual, contribuye al aprendizaje significativo de estos conceptos, en comparación con una metodología tradicional de transmisión y asimilación verbal de los conocimientos. Se realizó con 30 estudiantes de grado décimo del colegio Distrital Benjamín Herrera, ubicado en la zona dieciséis de Bogotá.

Metodología: la investigación es de tipo cuasiexperimental, consta de un grupo experimental y un grupo control de estudiantes de décimo grado. Con el primer grupo se implementó una estrategia basada en un software educativo basado en el modelo de aprendizaje por investigación; y mientras que al segundo se le enseñó mediante una estrategia tradicional. La información se recolectó por medio de dos instrumentos que determinaban las ideas previas y los errores conceptuales de los alumnos sobre los conceptos implicados, que se aplicaron antes y después de la estrategia; así mismo, se examinaron los reportes que el software Rived muestra de cada una de las actividades realizadas por los estudiantes para determinar la evolución de aprendizaje, y se tomaron en cuenta las observaciones anotadas en un diario de campo.

Conclusiones: los estudiantes del grupo control no construyeron un nuevo significado de los conceptos de punto de ebullición y densidad. Una vez finalizada la estrategia de transmisión de conceptos, presentaron las mismas relaciones conceptuales en su estructura cognitiva después de la instrucción y, además, la expresión de algunas que corresponden a un aprendizaje memorístico y repetitivo, sin comprender los entramados conceptuales en cuestión.

Al terminar la implementación del software, los estudiantes del grupo experimental presentan un aprendizaje significativo de los conceptos de punto de ebullición y densidad, dado que en la mayoría del grupo se observan procesos de reconstrucción de los entramados conceptuales al presentar un cambio de las ideas previas por un número amplio de relaciones conceptuales correctas sobre los temas en cuestión.

La estrategia con el software educativo basada en el modelo de enseñanza y aprendizaje por investigación es más eficiente que una estrategia de transmisión y asimilación de conceptos en el logro del aprendizaje significativo del concepto de punto de ebullición. El grupo experimental mostró un resultado del $53,33 \%$ más de relaciones conceptuales correctas que el grupo control en la aplicación postest, al concluir la implementación. 
La estrategia con software es más eficiente que una estrategia de transmisión asimilación de conceptos, en el logro del aprendizaje significativo del concepto densidad. El grupo experimental mostró un resultado de $60 \%$ mayor capacidad para establecer relaciones conceptuales correctas, que el grupo de control en la aplicación postest, al concluir la implementación.

El software Rived es una propuesta innovadora en la incorporación de los Ntic a la enseñanza de la química con un enfoque mediacional constructivo que permite de una manera eficaz el logro del aprendizaje significativo de los conceptos de punto de ebullición y densidad.

El software en mención permite el conflicto cognitivo y la reconstrucción conceptual, a partir de las ideas previas de los estudiantes sobre los atributos de criterio de los conceptos de punto de ebullición y densidad, y facilita la contrastación de dichas ideas en los laboratorios virtuales y en el análisis de datos.

El software Rived demuestra ser adecuado para trabajar a nivel individual o en parejas de estudiantes, permitiendo la colaboración entre los grupos a lo largo de la realización de las actividades.

Dicho programa es un instrumento de evaluación y mejoramiento de la enseñanza pues muestra los reportes de los estudiantes, lo que permite la realización de actividades de socialización en las que pueden aclararse dudas y dificultades aún no superadas en la interacción con el programa.
El software Rived es una herramienta ideal para la enseñanza de los conceptosde punto de ebullición y densidad, sin dejar de un lado la labor orientadora del profesor en algunos momentos del proceso de enseñanza.

\section{Desarrollo y evaluación de competencias en química}

\section{Martha Cecilia Santafé}

Descripción: esta es una propuesta que invita al estudiante a reflexionar sobre su proceso de formación académica, llevándolo a comprender que está inmerso en un contexto que evalúa a diario sus aprendizajes según sea la forma como se desempeñe en el mismo. Fue desarrollada con estudiantes de grado undécimo, promoción 2004, del Instituto Diversificado Albert Einstein, IDAE, de Mosquera, Cundinamarca, Colombia.

A partir de la concepción de evaluación identificada en los estudiantes como actividad final y cuantitativa del proceso enseñanza-aprendizaje, y de la manifestación de no conocer las competencias específicas del área de ciencias que se espera sean desarrolladas por ellos en el proceso educativo, se realiza un trabajo de reflexión y discusión al respecto empleando posteriormente programas guía de actividades (PGA) que permitan entender y utilizar la evaluación por competencias como herramienta reguladora de su aprendizaje; de esta forma, se va desarrollando la temática que nos interesa en el momento: la identificación de compuestos orgánicos. Además, las actividades propuestas han sido diseñadas con la intención de desa- 
rrollar las competencias propias del área de ciencias naturales y de evidenciar dicho desarrollo a través de desempeños específicos para cada competencia.

El diseño de las actividades se realizó con base en las competencias definidas por el Icfes como propias del área para interpretar situaciones, establecer condiciones, establecer hipótesis y regularidades, y valorar el trabajo en ciencias. El proceso de evaluación llevado a cabo comprendía, a la par con los programas guía, unas etapas claves que permitían al estudiante identificar sus dificultades y establecer estrategias a seguir para superarlas, como: evaluación inicial, evaluación reguladora, evaluación final y evaluación integradora.

Metodología: el desarrollo metodológico de la propuesta consta de varios instrumentos de apoyo utilizados para la recolección de información y para la orientación de cada sesión de clase.

Inicialmente, se identificó la concepción de evaluación con el IC1 (instrumento de caracterización 1) y el concepto de competencia manejado por los estudiantes con el IC2 (instrumento de caracterización 2), instrumentos aplicados de forma pre y post para contrastar la información inicial con la obtenida después de la aplicación de dos programas guía de actividades dirigidos al desarrollo de competencias.

A la par con el desarrollo de los programas guía de actividades, cada estudiante diligenció un instrumento de seguimiento general (ISG) que se convirtió en su carta de navegación para hacer de la evaluación una actividad reguladora del proceso educativo.

Conclusiones: en la medida que el estudiante ve la evaluación como actividad reguladora del proceso educativo, pierde el temor a ser evaluado y la aprovecha en términos de oportunidad para aprender. La valoración cuantitativa pasa a un segundo plano, siendo más importante el aprender y el aprender a superar las dificultades identificadas. Un proceso de este tipo requiere también un alto nivel de compromiso y autonomía por parte del estudiante, ya que tiende a particularizar el proceso, mientras que en grupos grandes de estudiantes se dificulta por el tiempo requerido para ello.

Cuando el estudiante conoce la forma como será evaluado y es consciente de las competencias que debe desarrollar, participa de forma más activa y consciente y, por tanto, obtiene buenos resultados en la evaluación final y en la generalizadora.

Cuando el estudiante supervisa su trabajo, se convierte en el primer evaluador. El seguimiento de cada una de las etapas de la evaluación, inicial, reguladora, final e integradora, permitió que el grupo representativo de estudiantes se apropiara de su capacidad frente al desarrollo de competencias para autorregular el proceso. Además, los estudiantes adquieren mayor seguridad en procesos de coevaluación y heteroevaluación. 


\section{Fuentes culturales del conocimiento químico}

Isabel Mercedes Torres Garay

Descripción: en esta investigación se intentó realizar una comprensión de las ideas y actuaciones de los estudiantes a partir de la interrelación entre conocimiento y cultura, dada y la importancia que se le debe dar a los conocimientos cotidiano, escolar y científico. Desde esta perspectiva, lo que se buscó fue identificar las fuentes culturales del conocimiento científico escolar, para promover el cambio conceptual en la noción de sustancias desde las ATAs con una perspectiva basada en la resolución de problemas. Para esto, se hizo necesario concebir el conocimiento desde un enfoque cultural, en el que no se entiende éste como una propiedad individual, sino que su validez se halla mediada por ser compartido por grandes grupos, como resultado de las negociaciones sociales con las ideas y constructos. Lo anterior proporciona percepciones e imágenes a sus miembros sobre el mundo que los rodea, esto es, representaciones colectivas que permiten establecer cómo participa la cultura en las diferentes formas intelectuales o conocimientos: ciencia, conocimiento común, concepciones inducidas, que se derivarían del entorno sociocultural de los estudiantes y, en particular, del lenguaje y la cultura.

Por esta razón, el problema de investigación estaba encaminado a determinar la incidencia de las fuentes culturales del conocimiento en el cambio conceptual de la noción de sustancia, a partir de las
ATAs, desde la perspectiva de la resolución de problemas. En este sentido, los objetivos planteados estaban dirigidos por una parte hacia la identificación de las fuentes culturales de los que proviene el conocimiento químico de los estudiantes y la determinación del papel mediador del contexto sociocultural en los procesos de construcción del conocimiento escolar de aquellos. Y, por otra parte, hacia la promoción de un eventual cambio conceptual en la noción que los estudiantes poseen de sustancia desde las ATAs, con la perspectiva de la resolución de problemas.

Metodología: la metodología de esta investigación puede incluirse dentro del enfoque etnográfico e interpretativo, porque intentó mostrar el estado presente de las fuentes culturales del conocimiento químico de los estudiantes en el momento de la investigación y el estado posterior de estas, luego de la intervención pedagógica. Así, en un primer momento, se exploraron las fuentes culturales del conocimiento químico de los estudiantes, en relación con la noción de sustancia, mediante un proceso inductivo que partió de sus formas de explicación, para llegar luego a la identificación, interpretación y categorización de las fuentes culturales, y posteriormente, al análisis del posible impacto de la intervención pedagógica en la búsqueda de un cambio conceptual de los estudiantes.

El proceso de la investigación se adelantó en las siguientes fases: revisión documental de los supuestos teóricos del abordaje pedagógico para la elaboración de los antecedentes; diseño y aplicación 
del instrumento pretest; intervención pedagógica desde las ATAs con una perspectiva de resolución de problemas; aplicación del instrumento postest, y procesamiento, análisis e interpretación de los resultados.

Conclusiones: algunos de los resultados de esta investigación muestran que en los ambientes escolares se le da un gran énfasis a la justificación de las teorías científicas, sin prestar ningún interés a los contextos de descubrimiento del conocimiento. Las explicaciones dadas por los estudiantes, en el instrumento pre test, se aproximan a una visión superficial del mundo, esto es, no iban más allá de las evidencias, sino que realizaban inducciones a partir de las observaciones sensibles. De tal manera, concebir al ámbito escolar como circunscrito en ámbitos más amplios (una sociedad, una cultura) haría posible entender que los contenidos de las teorías y conceptos corresponden a perspectivas y concepciones de las sociedades en las que ellas se formulan, y que por procesos de transposiciones de unos ámbitos a otros, los conceptos y teorías dan origen a otros. Además, que esta transposición está determinada por criterios ideológicos, es decir, en el caso de la noción de sustancia, la transposición al ámbito escolar trae consigo criterios ideológicos del ámbito en el que se origina.

Los resultados muestran, además, que los estudiantes se apropian y utilizan las fuentes culturales del conocimiento para legitimar las versiones en las que creen, tal y como se apreció en el primer momento de la prueba pretest y en el segundo momento de la prueba posttest. Con estas ideas y actuaciones, ellos construyen su lugar en la experiencia escolar como sujetos activos, con conocimientos que son validados, creencias, valores, diversas maneras de decir, autonomía y responsabilidad frente a situaciones concretas.

Los resultados de la investigación mostraron la existencia de cuatro fuentes de conocimiento: analógica, raciocinio, causal y teleológica, que provienen tanto del mundo cotidiano, como de la imagen de ciencia concebida como una actividad social, es decir, de la consideración que tienen los estudiantes, en torno al saber científico, como un cuerpo de conocimientos, patrimonio absoluto de científicos e investigadores. Tradicionalmente se ha considerado que la escuela le brinda al estudiante conocimiento científico. Sin embargo, en este trabajo se considera la experiencia escolar como el conocimiento escolar que está determinado por todas las fuentes culturales del conocimiento encontradas, además del conocimiento científico escolar (imagen de las ciencias naturales en el contexto escolar), como del mismo conocimiento cotidiano (experiencial cotidiano).

Se puede concluir, en relación a la promoción del cambio conceptual en los estudiantes, que si se concibe el cambio conceptual no sólo como el que tiene que ver con operaciones intelectuales (clasificar, ordenar, comparar, etc.), sino que también se le aplica una atribución de sentido y significado, es decir, una asimilación vital, empática y motivacional, el cambio conceptual sólo se hace posible cuando en la mente se tiene una imagen 
mental mínima que permite asignar un marco de referencia a ese algo que se intenta asimilar al esquema interpretativo propio. Esta representación mental se refiere a la forma como, con base en las prácticas sociales e interpretativas de cada grupo social, se disponen y se organizan las estructuras de las cosas. En este sentido, el cambio conceptual caliente implica establecer relaciones y confrontaciones entre diversas formas de representación -básicamente entre la representación que posee el estudiante con la representación científica que intenta presentar el profesor-.

En este sentido, la articulación de lo planeado con lo ejecutado en el aula, la retroalimentación que se observó en todo el proceso entre las diferentes actividades propuestas y la forma como concuerdan estas actividades con las preguntas problematizadoras de los estudiantes permiten afirmar que la intervención pedagógica formulada para esta investigación es válida no sólo para promover un cambio conceptual caliente, sino que se convirtió en el insumo necesario para dar cuenta de las fuentes culturales del conocimiento químico de los estudiantes y que, a partir de un encadenamiento de un ambiente de clase propicio, es posible recuperar la experiencia como una forma más de acercarse al mundo natural.

\section{Modelo de enseñanza y aprendizaje por investigación para el desarrollo de la argumentación y el aprendizaje significativo de reacción y ecuación química}

Claudia Patricia Posada González

Descripción: la investigación se realizó con estudiantes de grado undécimo del colegio distrital Nuevo Monteblanco, ubicado en la zona quinta de Usme en Bogotá. El objetivo central fue generar el aprendizaje significativo de los conceptos reacción y ecuación química, y desarrollar la argumentación en los estudiantes por medio del diseño, aplicación y evaluación de un programa guía de actividades.

Esta propuesta se fundamenta en el proceso de producción del conocimiento, ya que en él convergen la autonomía, el pensamiento divergente, la experimentación y argumentación propias de las ciencias. Al implementar una estrategia pedagógica y didáctica de enseñanza aprendizaje por investigación, a partir de un programa guía de actividades para la resolución de situaciones problemáticas, se desarrolla la argumentación propia de la química y el aprendizaje significativo de los conceptos reacción y ecuación química.

Metodología: pra desarrollar el presente trabajo de investigación se utilizó un instrumento diagnóstico para determinar los preconceptos y nivel de argumentación de los estudiantes; éste se aplicó antes de desarrollar la estrategia didáctica y al finalizar ésta. Se aplicaron 16 instrumentos durante el transcurso del programa guía de actividades. 
Conclusiones: los estudiantes desarrollaron la competencia argumentativa, porque en la mayoría de los textos escritos durante el proceso de resolución de las situaciones problémicas se refleja la naturaleza argumentativa al dejar entrever mayor estructuración, coherencia química e intencionalidad al expresar sus puntos de vista, acuerdos y conclusiones de las situaciones problémicas.

En el proceso de enseñanza y aprendizaje, como en este caso la argumentación, los conceptos, la reacciones y ecuaciones químicas, es necesario una regulación continua de la enseñanza, porque el docente debe identificar las necesidades del estudiante y proporcionarle herramientas para ayudarle en el aprendizaje, porque el estudiante ha de aprender a reconocer sus dificultades, encontrar caminos para superarlos. Este proceso se desarrolló mediante el modelo de enseñanza por investigación.

El programa guía constituye una forma de orientar el aprendizaje de los estudiantes por medio del diseño, aplicación y evaluación de actividades organizadas de forma progresiva en conceptos y dificultades, pero también es una forma de orientar el control del tiempo y hacer las cosas de una forma más eficaz y eficiente para el proceso de enseñanza y aprendizaje de la química.

En los estudiantes se generó el aprendizaje significativo de reacciones y ecuaciones químicas, desde la propuesta de Ausubel, porque se evidenció en ellos la diferenciación progresiva de estos conceptos a través de la construcción de los ma- pas conceptuales y el análisis, desarrollo y solución de las situaciones problémicas.

En el aprendizaje de la química es muy importante la discusión y el contraste de ideas, lo que se observa en la aplicación de la metodología del trabajo científico para dar solución a cada una de las situaciones problemáticas planteadas, proceso que facilita el contraste de ideas y el uso del lenguaje cotidiano combinado con una argumentación clara y coherente, utilizando los conceptos relacionados con reacción y ecuación química, para que el lenguaje se transforme en una argumentación propio de la química. Los estudiantes desarrollan la argumentación convincente a través del programa guía de actividades y no hacen uso de la argumentación lógica y científica.

\section{Maestría en \\ Docencia de las Matemáticas}

Validación del modelo del profesor Federici sobre la construcción de los números racionales positivos: obstáculos didácticos en la relación parte-todo

\section{Carmen Andrade Escobar}

Descripción: este documento es el resultado de una investigación sobre la construcción de los números racionales en séptimo grado. Los antecedentes señalan que las dificultades de los estudiantes para dar sentido a los números racionales tienen su origen en la enseñanza de la fracción como relación parte-todo. La fracción tiene como base el número contador, pero el número racional es una relación. Esta falta de claridad en el sig- 
nificado de los números ha conducido a planteamientos estrechos o ficticios en la enseñanza. El problema es que se hace énfasis en la sintaxis de los números. El modelo del profesor Federici aporta a la didáctica de las matemáticas sobre la construcción de los números racionales desde su semántica, como medida entre valores de una magnitud; idea básica para la construcción de las matemáticas y de la ciencia de los años superiores.

Este trabajo consta de tres capítulos. En el primer capítulo se presenta la delimitación del problema, que consiste en antecedentes de investigaciones sobre los números racionales y la fracción, justificación, definición del problema, hipótesis y objetivos. El capítulo dos comprende el marco teórico de la investigación: se estudia el proceso filogenético del número; el tratamiento de los números racionales en los textos; el modelo Federici sobre la construcción de los racionales; los obstáculos didácticos que se presentan en los textos, y criterios de aprendizaje. El capítulo tres consta del procedimiento en la aplicación del modelo en tres etapas: descripción de la población, organización del modelo de enseñanza y análisis de resultados. Por último, se presentan las conclusiones y recomendaciones, la bibliografía y los anexos.

Metodología: la metodología consistió en definir unas etapas en la construcción de los números racionales con base en el modelo del profesor Federici. Estas etapas surgen de los resultados del análisis del proceso filogenético del número $y$, desde luego, su presentación se aco- ge al modelo en estudio. Posteriormente, se diseñaron las sesiones acorde a cada etapa, pero con la posibilidad de ampliar o cambiar este orden en respuesta a las necesidades del grupo de referencia. Se procedió a clasificar el grupo de referencia en cinco subgrupos, de acuerdo con su nivel académico en matemáticas y su motivación. De cada grupo se escogió un estudiante al azar para tomarlo como estudio de caso. Inicialmente, se realizó una prueba con el fin de determinar los conocimientos previos sobre los números racionales, específicamente, sobre cómo plantear y resolver problemas, y el significado dado a los números racionales. La aplicación tuvo dos etapas, una en sexto grado, aproximadamente durante mes y medio, y la segunda al inicio del curso de matemáticas en séptimo grado, aproximadamente durante cuatro meses. Al final de esta segunda etapa se realizó otra prueba con el fin de evaluar los avances. Por último, se realizó una prueba tres meses después para confirmar estos avances.

Conclusiones: los avances de los estudiantes, de séptimo grado, en la construcción semántica de los números racionales a partir del modelo del profesor Federici invitan a pensar en este modelo como la alternativa para la didáctica de los números racionales, porque se evitan los obstáculos didácticos y, además, porque es una base sólida para el estudio de las matemáticas y de la ciencia de los años superiores. La enseñanza de la fracción, como la relación entre la parte y el todo, es un impedimento para superar el obstáculo epistemológico entre los naturales y los 
racionales, porque conceptualmente se ha visto que es diferente al número racional. El estudio de los racionales con operadores sobre longitudes, específicamente sobre segmentos, es una herramienta didáctica para construir un número con un significado diferente al número contador. El estudio de los sistemas numéricos organizado de acuerdo al orden de cerradura de las operaciones aritméticas, N, Z, Q, R, C2, C4, no es para los niños sino para las computadoras. El orden histórico $\mathrm{N}, \mathrm{Q}+$, Q-, R, C2, C4 sigue un proceso acorde con un contexto sociocultural, que va de lo pragmático a lo teórico y es más acorde para la didáctica de las matemáticas.

\section{Organizaciones didácticas matemáticas y criterios de evaluación en torno a la multiplicación}

\section{Rodolfo Vergel Causado}

Descripción: investigación cualitativa interpretativa, que consiste en responder la pregunta: ¿Son los criterios de evaluación del aprendizaje de la multiplicación en las aulas de tercero, cuarto y quinto de la educación básica dependientes de las organizaciones didácticas matemáticas (ODMs) de los contenidos relativos a este concepto? El objetivo del estudio fue describir y analizar cómo las organizaciones didácticas matemáticas de los contenidos relativos a la multiplicación constituyen un factor determinante de los criterios para valorar su aprendizaje.

Para la consecución de este objetivo se hace necesario: describir y analizar la organización didáctica matemática (ODM) de los contenidos relativos a la multiplica- ción en cada uno de los grados tercero, cuarto y quinto de la educación básica en tres colegios de Bogotá; caracterizar los criterios de evaluación del aprendizaje de la multiplicación en los grados tercero, cuarto y quinto de la educación básica en dichos colegios; establecer la relación de dependencia entre los criterios de evaluación caracterizados y las organizaciones didácticas matemáticas de los contenidos relativos a la multiplicación.

Metodología: para ejecutar el objetivo general: describir y analizar cómo las ODMs de los contenidos relativos a la multiplicación constituyen un factor determinante de los criterios para valorar su aprendizaje y, en consecuencia, intentar responder la pregunta de investigación: ¿Son los criterios de evaluación del aprendizaje de la multiplicación en las aulas de tercero, cuarto y quinto de la educación básica dependientes de las organizaciones didácticas matemáticas de los contenidos relativos a este concepto?, se articulan los dos siguientes focos de investigación: Criterios de evaluación del aprendizaje de la multiplicación (F1) y Estudio de la dependencia de los criterios de evaluación del aprendizaje de la multiplicación en las aulas de tercero, cuarto y quinto de la educación básica de las ODMs de los contenidos relativos a este concepto (F2). Cada foco se desglosa en una serie de subobjetivos subsidiarios del objetivo general, y para la operativización de estos subobjetivos se plantean cuestiones específicas de investigación que constituyen subpreguntas de la pregunta general de investigación. 
Los niveles de investigación exigen la definición de unidades de análisis para poner de presente el carácter dependiente de los criterios de evaluación del aprendizaje de la multiplicación de las ODMs de los contenidos relativos a este concepto. Las unidades de análisis son:

Para el primer foco: las preparaciones de clase en las aulas de tercero y cuarto; las sesiones de clase de tercero, cuarto y quinto; las pruebas escritas propuestas por el profesor en el aula; las respuestas dadas por el profesor a la entrevista semiestructurada en relación con los aspectos evaluativos; y el libro de texto utilizado por la profesora del grado tercero.

Para el segundo foco: las preparaciones de clase en las aulas de tercero y cuarto; el plan de aula institucional del grado tercero; las sesiones de clase de los grados tercero, cuarto y quinto; y las respuestas a la entrevista semiestructurada en relación con los aspectos de organización de los contenidos matemáticos en torno a la multiplicación. Posteriormente, se plantean las categorías con base en el marco teórico, para intentar responder a la pregunta de investigación formulada en este estudio.

Conclusiones: con base en el análisis e interpretación (apartado 3.4), se puede afirmar que en las aulas estudiadas efectivamente las organizaciones didácticas matemáticas de los contenidos relativos a la multiplicación constituyen un factor determinante de los criterios para valorar su aprendizaje.

\section{Aproximación al concepto de nú- mero real con estudiantes de grado octavo de básica secundaria \\ Wilson Gordillo Thiriat \\ Mario Eduardo Thiriat Rojas}

Descripción: este trabajo presenta una propuesta didáctica para la enseñanza del concepto de número real, como una opción diferente al tratamiento formal y riguroso que tradicionalmente se usa para abordar este concepto, correspondiendo a un estudio de réplica parcial de la tesis doctoral de Isabel Romero Alvadalejo: "La introducción al número real en enseñanza secundaria: una experiencia de investigación-acción", ya que se ha tomado todo el diseño y la mayor parte del proceso metodológico de investigación que la doctora Romero presenta en su tesis.

Con respecto a la rigurosidad que exige la enseñanza del programa de las llamadas matemáticas modernas, se proponen los siguientes interrogantes:

1. ¿El tratamiento convencional formal basado en las llamadas matemáticas modernas que se viene desarrollando para introducir los números reales en la secundaria, permite al estudiante alcanzar el nivel de pensamiento numérico esperado por la sociedad, que ha formulado objetivos, logros, competencias y estándares para este nivel?

2. ¿Tiene suficiente significado para los estudiantes el tratamiento didáctico de introducción al concepto de número real fundamentado en la formalidad de las matemáticas modernas? 
Interrogantes que en principio se abordan elaborando una prueba diagnóstica cuyos resultados evidenciaron en los estudiantes poca profundidad conceptual del objeto ideal "número", así como la falta de aplicación de procesos algorítmicos que involucran los cambios de representación (simbólicas y gráficas), por lo que se plantea la posibilidad de una propuesta didáctica caracterizada por la complejidad del concepto de número real y la interacción entre los tipos de representaciones digitales (simbólicas) y analógicas (gráficas). Propuesta que se formula y categoriza desde un análisis para la organización del contenido, teniendo en cuenta el desarrollo histórico para la construcción del concepto de número real y en el que se determinan dos focos de investigación para conseguir el objetivo general del trabajo que se hace realizable, a través de unas sesiones de clase en las que se va analizando la comprensión del contenido, dando validez a la hipótesis que se formula en términos de la viabilidad de esta propuesta didáctica.

Metodologa: esta investigación se enmarca dentro de la metodología de investigación-acción, conformada por las siguientes fases fundamentales: planificación, acción, observación y reflexión, así como su secuencialidad en forma de ciclos. Particularmente, el proceso de investigación inicia a partir de un instrumento diagnóstico que se aplica a los estudiantes del grado 805 del Colegio Mayor de San Bartolomé. El instrumento presentó los resultados que se muestran en la fase de acción, resultados que per- mitieron realizar la planificación de la propuesta didáctica acorde con las necesidades detectadas al revisar los resultados de dicho instrumento.

En la fase de planificación, para recopilar la información con el fin de escribir la propuesta, se opta por seguir el modelo propuesto en la tesis doctoral de Isabel Romero, teniendo presente el análisis y desarrollo histórico-crítico del concepto de número real, así como las condiciones evolutivas y académicas de nuestros estudiantes. Para cada sesión de clase, y específicamente para cada observación, se tuvieron en cuenta los siguientes instrumentos de observación:

- Producción escrita realizada por el estudiante.

- Tipo de solución planteada por los estudiantes a los ejercicios propuestos.

- Participación y aportes del grupo para el desarrollo de la sesión de clase.

Se optó por hacer una videograbación del trabajo desarrollado por los estudiantes; se cerró el Trabajo de investigación con el instrumento llamado postest, que mostró los resultados que se presentan tanto en la fase de acción como en la de reflexión.

Conclusiones: el diagnóstico se formalizó a partir de un instrumento de observación (pretest) que reveló poca profundidad conceptual del objeto matemático ideal "número", así como la falta de aplicación de procesos algorítmicos que involucran los cambios de representación digital (simbólica) y ubicación de números racionales sobre la recta numérica (representaciones analógicas), lo que 
ubicaba a los estudiantes en un proceso de desarrollo de pensamiento de bajo nivel, con respecto a los números reales; al mismo tiempo, nos llevó a plantear la necesidad de diseñar la propuesta didáctica en el trabajo procedimental con números racionales, acorde con la organización de contenidos de la propuesta curricular del Ministerio de Educación Nacional de Colombia, publicada en 1988.

Con el transcurrir de las sesiones de clase se consolidó el desarrollo de la propuesta didáctica, que en un principio presentó dificultades debido a la actitud mostrada por el grupo en cuanto a la falta de interés en la asignatura, reflejada en el poco trabajo fuera de la clase y en las manifestaciones de algunos estudiantes para que la clase se desarrollara de forma tradicional (explicación de contenidos, ejemplos y ejercicios).

En la propuesta desempeñaron un papel fundamental los distintos sistemas de representación de los números reales: el sistema de notación decimal y la notación operatoria de los reales, dentro de las notaciones simbólicas, y el modelo de la recta, junto con la medida de longitudes en el terreno de las representaciones gráficas.

En cuanto a la identificación y el manejo de sistemas de representación, nuestros estudiantes mostraron comprensión del significado de los dígitos en el sistema de notación decimal y algunas dificultades a la hora de interpretar signos gráficos. Así, pudimos observar la resistencia de varios estudiantes a considerar como equivalentes representaciones de los números reales que presentan face- tas distintas y exclusivas de cada una. No todos los estudiantes parecen dispuestos a admitir esto de buen agrado, aunque el hacerlo facilita el paso siguiente de la concreción de conceptos como el de número real. 